\title{
Cumulative Meta-Analysis for Genetic Association: When Is a New Study Worthwhile?
}

\author{
Michael A. Rotondi ${ }^{\mathrm{a}}$ Shelley B. Bull ${ }^{\mathrm{b}}$ \\ ${ }^{a}$ School of Kinesiology and Health Science, York University, and ${ }^{b}$ Samuel Lunenfeld Research Institute, \\ Mount Sinai Hospital, Dalla Lana School of Public Health, University of Toronto, Toronto, Ont., Canada
}

\section{Key Words}

Sample size and power $\cdot$ Sequential design $\cdot$ Statistical genetics

\begin{abstract}
Objectives: In this paper, we address the questions: how large a sample size would be required to show genome-wide significance between a single nucleotide polymorphism (SNP) and a genetic trait in a meta-analysis of a newly planned study together with the existing ones? Or alternatively: will a planned study of size $n$ be able to provide evidence of a genetic association when this study is combined with a current meta-analysis? Methods: We examine the potential impact of a newly planned genetic study on an existing metaanalysis through the use of a simulation-based algorithm. The proposed approach provides an empirical estimate of the power of the updated meta-analysis to detect genomewide significance $\left(p<5.0 \times 10^{-8}\right)$ of a complex trait and each of a set of specific SNPs of interest or the expected $p$ value of the updated meta-analysis including the current and proposed studies. Results: This technique is illustrated in the context of an updated meta-analysis of case-control studies in Paget's disease. A second example illustrates the impact of adding a newly planned study to a large meta-analysis of
\end{abstract}

SNP associations with human height. Conclusions: The proposed algorithm is particularly useful for the design of studies to assess a selected set of high-priority SNP associations that are 'nearly' significant in meta-analysis of existing studies. The results may help investigators decide whether an updated meta-analysis is likely to achieve genome-wide significance.

Copyright $\odot 2012$ S. Karger AG, Basel

\section{Introduction}

As the potential effects of many single nucleotide polymorphisms (SNPs) are relatively small, increasingly large sample sizes are required to detect a statistically significant association between a SNP and a phenotype of interest [1]. This has led to numerous trait- and disease-specific consortia in an effort to increase available sample size and thereby improve the ability to detect smaller associations through the meta-analysis of multiple genome-wide association study (GWAS) data.

Investigation of the genetics of complex traits has typically proceeded from the examination of genetic association within a specific population to the accumulation of evidence from multiple studies in various populations.

\section{KARGER}

Fax +4161306 1234

E-Mail karger@karger.com

www.karger.com
(C) 2012 S. Karger AG, Basel

0001-5652/12/0742-0061\$38.00/0

Accessible online at:

www.karger.com/hhe
Michael A. Rotondi

School of Kinesiology and Health Science

York University, 4700 Keele Street

Toronto, ON M3J 1P3 (Canada)

E-Mailmrotondi@yorku.ca 
In this report, we propose a technique to assess the impact of adding a newly planned genetic association study to a cumulative meta-analysis of available study data. It may be applied in situations where there is suggestive evidence of a statistically significant association between a SNP and a trait of interest; however, the strength of this association has not achieved strict genome-wide significance levels. Because the technique builds on existing meta-analysis data for a specific SNP association, this approach would be most useful when the investigator is interested in a selected set of SNPs that have suggestive evidence of statistical significance, or perhaps have a known biological mechanism. Moreover, this technique may be of particular use in a consortium setting, where data are shared with other investigators after initial publication of study results. The method involves modifications of an approach developed by Sutton et al. [2] and recently extended to the case of cluster randomized trials [3]. In this report, we extend the method to the setting of a planned genetic association study. For specificity, we initially assume that the planned study has a case-control design, and there is a particular candidate SNP association of interest. Our presentation focuses on the challenges unique to this application and provides a brief review of the relative merits of the fixed and random effects meta-analysis models in genetic association studies.

Briefly, the approach is summarized as follows: for a given phenotype, the investigator selects a candidate SNP (or set of SNPs). A preliminary meta-analysis of this information is then used to generate an estimate of the distribution of potential results of the newly planned study. Individual-level data are generated according to this potential genetic effect and range of plausible values, and summary statistics for the 'new study' are obtained. Two features of the proposed method distinguish it from standard power calculations. First, it incorporates information about effect size estimates and standard errors from each of the existing studies available for meta-analysis, as well as the observed heterogeneity in sample sizes and effect estimates between studies. Second, it allows for uncertainty in knowledge about the effect sizes and minor allele frequencies expected in the newly-planned study that will be added to the existing meta-analysis. The algorithm is then replicated a large number of times. Repeating this approach for various study sizes allows the investigator to obtain an empirical estimate of the anticipated $p$ value or power of the revised meta-analysis to detect a statistically significant association between the SNP and trait of interest.

A detailed outline of the proposed method, and preliminary information regarding the fixed and random ef- fects meta-analysis models follows. To illustrate its particular strengths, the proposed algorithm is applied to two recent meta-analyses, one in Paget's disease [4] and a second in human height [5]. Appropriate R code to implement the sample size estimation algorithm is available in the software package metaSNPPER which is freely available from the R project [6].

\section{Methods}

\subsection{The Evidence-Based Algorithm}

An outline of the proposed procedure is presented in table 1. This is adapted from Sutton et al. [2] and is summarized in the context of a planned case-control study. Section 3.2 describes the adaptation to a quantitative trait.

\subsection{Meta-Analysis Preliminaries}

We consider the following meta-analysis model for the combination of $K$ case-control studies as adapted from Lin and Zeng [7]. For the analysis of each SNP, consider the data pairs $Y_{k i}, X_{k i}$, where $Y_{k i}$ is the disease status $(1=$ case, $0=$ control $)$ for the $i$-th participant of the $k$-th study, and $X_{k i}$ is the corresponding genotype. The genotype may be coded by the values of 1 or 0 , respectively, depending on the postulated genetic model: dominant ( 1 if any minor allele is present; 0 otherwise) or recessive ( 1 if the individual has two copies of the minor allele; 0 otherwise). Alternatively, an additive genotype model may be used, whereby the genotype score corresponds to the number of minor alleles at the SNP $(0,1$ or 2 , respectively).

We assume the classical logistic regression model:

$$
\operatorname{Pr}\left(Y_{k i}=1\right)=\frac{\exp ^{\alpha_{k}+\beta_{k} X_{k i}}}{1+\exp ^{\alpha_{k}+\beta_{k} X_{k i}}}
$$

where the $\alpha_{k}$ are study-specific intercepts, and $\beta_{k}$ is the log odds ratio representing the genetic effect in each of the $K$ studies. Let $v_{k}$ denote the estimated variance of the estimated log odds ratio, $\hat{\beta}_{k}$.

Within this framework, the fixed effect meta-analysis estimate of $\beta$ is obtained from:

$$
\hat{\beta}_{F}=\frac{\sum_{k=1}^{K} \omega_{k} \hat{\beta}_{k}}{\sum_{k=1}^{K} \omega_{k}}
$$

where $\omega_{\mathrm{k}}=\nu_{k}^{-1}$. Assuming the sample size from each of the $K$ studies is relatively large, so that we may consider these variances known, $\hat{\beta}_{F}$ has an asymptotic normal distribution centered around $\beta$ with variance

$$
\left(\sum_{k=1}^{K} \nu_{k}^{-1}\right)^{-1} \text {. }
$$

This information is incorporated into the prediction of the new effect size in the third step of the algorithm (table 1).

In contrast to the fixed effects model, the presence of betweenstudy variation is explicitly acknowledged within the random ef- 
fects framework. In essence, this model can be informally derived by placing an additional condition on the observed $\beta_{k}$, namely that these are sampled from a normal superpopulation of effect measures centered around $\beta$ with variance $\tau^{2}$. The introduction of this additional assumption allows inclusion of the betweenstudy variance parameter $\tau^{2}$ in the meta-analytic study weights. Although the random effects estimate of the pooled effect measure is constructed in the same manner as (2), the study weights, $\omega_{k}$, must now account for the between-study heterogeneity, $\tau^{2}$. Hence, let $\omega_{k}^{\prime}=\left(\nu_{k}+\hat{\tau}^{2}\right)^{-1}$ denote the random effects weights, and

$$
\hat{\beta}_{R}=\frac{\sum_{k=1}^{K} \omega_{k}^{\prime} \hat{\beta}_{k}}{\sum_{k=1}^{K} \omega_{k}^{\prime}}
$$

This model poses the additional task of estimating $\tau^{2}$. The classical approach [8] relies on using the statistic

$$
Q=\sum_{k=1}^{K} \omega_{k}\left(\hat{\beta}_{k}-\beta_{F}\right)^{2}
$$

as a measure of between-study variation. Specifically,

$$
\hat{\tau}^{2}=\frac{Q-(K-1)}{\sum_{k=1}^{K} \omega_{k}-\left(\sum_{k=1}^{K} \omega_{k}^{2}\right) / \sum_{k=1}^{K} \omega_{k}} \text { if } Q>K-1
$$

and

$$
\hat{\tau}^{2}=0 \text { if } Q \leq K-1
$$

Note that in practice $\tau^{2}$ is typically estimated from a relatively small number of studies and may thus be subject to increased uncertainty as compared to the estimation of each $v_{k}$. For this reason, we assume that the $t$-distribution, centered around $\beta_{R}$ with $K-2$ degrees of freedom forms a reasonable basis for prediction of a new genetic effect [9]. This approach is required when the random effects meta-analysis model is incorporated in the second step of the algorithm (table 1).

A popular solution to the problem of heterogeneity is to quantify the amount of heterogeneity in the set of $K$ observed genetic effects. The commonly adopted approach is to include the $I^{2}$ statistic as presented by Higgins and Thompson [10]. This statistic is easily calculated from a current meta-analysis and has a natural range between zero and one, where between-study heterogeneity in the genetic effects increases as this statistic approaches one. For reference, this statistic can be obtained from the $Q$ statistic, as $I^{2}=(Q-K+1) / Q$.

In general, we support the examination of the fixed and random effects models when applying the approach in study design. In particular, the fixed and random effects estimates of $\beta$ (and their respective variances) may differ substantially from one another, leading to divergent findings. Note that in application, the fixed effects model typically yields greatest power to detect genome-wide significance and is typically less conservative than the random effects framework, since $\operatorname{Var}\left(\hat{\beta}_{F}\right) \leq \operatorname{Var}\left(\hat{\beta}_{R}\right)$. The $I^{2}$ value from meta-analysis of the existing data can inform the choice between fixed and random effects models, but the potential for heterogeneity in the new study data also needs to be considered. Moreover, for sample size estimation, it may be desirable to also consider the random effects model as a more realistic examina-
Table 1. Algorithm outline for a case-control study

1 Select a fixed number of cases, $n_{\text {cases }}$, and controls, $n_{\text {controls }}$, that are available for the newly planned study. Note that the approach does not require a balanced design.

2 Perform a meta-analysis of the existing genetic association data using a fixed or random effects model. This produces an estimate of the $\log$ odds ratio, $\hat{\beta}_{P}$ and its estimated variance, $\operatorname{Var}\left(\hat{\beta}_{P}\right)$ for each SNP. Note that an investigator may adjust $\hat{\beta}_{P}$, or increase $\operatorname{Var}\left(\hat{\beta}_{P}\right)$ in an effort to account for the 'winner's curse' [26].

3 Using the preliminary meta-analysis information, sample a new genetic effect from a $N\left(\hat{\beta}_{P}, \operatorname{Var}\left(\hat{\beta}_{P}\right)\right)$ or a $T$ distribution, depending on the selection of the fixed or random effects models.

4 Specify appropriate values for the rate of observing the candidate SNP variants in the control population (i.e. the MAF). This may be estimated from studies included in the existing meta-analysis, or by external reference information (e.g. Hapmap [29] or 1,000 Genomes [30]). Note that the use of multiple values in a sensitivity analysis is recommended in practice, as the MAFs may vary across study populations. Alternatively, prior distributions may be investigated to examine the role of variation of the MAF in the planned study.

5 Generate individual-level data according to the sampled genetic effect and the anticipated MAF (or range). A simple binomial model may be used to randomly generate the observed outcomes, $Y_{i}$, in the cases and controls using the observed genetic effect size. In this manner, we are adding two levels of variation, corresponding to the study and the individual levels.

6 Calculate summary measures of association, such as the log odds ratio and its estimated variance for the 'new' study.

7 Add the new study data to the existing meta-analysis, which is then re-meta-analyzed using either the fixed or random effects model.

8 Repeat steps 3 through 7 a large number of times $N$ (say $10,000)$, noting the outcome for the desired genome-wide significance criterion.

9 Repeat the entire procedure with a revised value of $n=n_{\text {cases }}$ $+n_{\text {controls }}$, thus examining the impact of alternative sample sizes on the combined meta-analysis of the previously completed and proposed study. Values of $n$ can be chosen from a set of plausible numbers of subjects that are available to the investigator. In particular, note that in many studies $n_{\text {controls }}>n_{\text {cases }}$, as previously genotyped controls may be available from other sources. In addition, should a very large study size be required, an investigator may conclude that they are unlikely to detect that this association is significant and choose to devote resources elsewhere. 
tion of the potential effect of the planned study in the presence of heterogeneity such as may arise due to population structure or differences in environmental background.

\subsection{Decision Rules}

2.3.1. p Value Approach

The algorithm thus performs a Wald-based hypothesis test on the cumulative meta-analytic estimate $\hat{\beta}_{P}$ using either the fixed or random effects model. In this manner, $\beta_{P}$ is the underlying parameter of interest, as it represents the cumulative knowledge of the genetic association, rather than the effect measure in a single, planned case-control study. Upon 'completion' of the planned study (table 1 , steps 5 and 6), the hypothesis test $H_{0}: \beta_{P}=0$ versus $H_{A}: \beta_{P} \neq 0$, is performed and the resulting $\mathrm{p}$ value is obtained. This value is retained for each iteration $(N)$. An empirical estimate of the anticipated p value when adding a study of size $n$ may be obtained by selecting the median $p$ value that is observed over all iterations. Use of the median $p$ value is recommended in practice as it is less sensitive to the potential extreme $\mathrm{p}$ values that may be observed. However, an investigator may also investigate the distribution of these $\mathrm{p}$ values to assess their variability.

\subsubsection{Power to Detect Genome-Wide Significance}

In a similar manner, the approach may be used to graphically examine the anticipated power of a subsequent meta-analysis to reach genome-wide significance $\left(<5.0 \times 10^{-8}\right)$ [11] when a study of size $n$ is added to an updated meta-analysis. The observed $\mathrm{p}$ value for the hypothesis test $H_{0}: \beta_{P}=0$ versus $H_{A}: \beta_{P} \neq 0$ is obtained as before. However, if the $\mathrm{p}$ value of this hypothesis test reaches genome-wide significance $\left(<5.0 \times 10^{-8}\right)$, this decision is noted. This entire procedure (table 1 , steps $3-7$ ) is repeated a large number of times $(N)$ and the empirical power to detect genomewide significance is estimated as the proportion of iterations where the desired significance level is obtained. This entire procedure is then repeated using other values of $n$, allowing calculation of appropriate power curves for the updated meta-analysis.

\subsection{Prior Distributions and Parameter Values}

In addition to the potential variation in the unknown genetic association of the planned study, uncertainty in the minor allele frequency (MAF) for the given SNP may also play a critical role in the observed effect size of the planned study. In the presence of relatively large studies, the examination of fixed values of the MAF may be reasonable, provided the planned study is taking place in a similar population. Alternatively, one can place prior distributions on the MAF to take account of the role of uncertainty in the MAF. In this case, a simulated value of the MAF is obtained from an appropriate distribution for each iteration, and this value is used in the fourth step of the algorithm (table 1). A suitable prior density for the anticipated MAF may be represented using a beta distribution. This distribution is appropriate for proportions as it is naturally bound within the interval $[0,1]$, while specification of parameter values, $\alpha$ and $\beta$ can provide a wide variety of density shapes [12]. Should the investigator have a reasonable basis for the plausible range of the MAF, a simple uniform distribution on that interval may be appropriate as an alternative. Furthermore, when a large number of estimates for the MAF are available, empirical density functions [13] may also be explored. This approach constructs a reasonable prior density using empirical smoothing techniques on the entire set of observed MAFs for the SNP of interest in the previously completed studies. The benefit of this approach is that an investigator need not consider explicit distributional constraints. Finally, note that as the technique only requires simulating data for a single study at each iteration, processing time is typically on the order of a few seconds to a few minutes in order to produce power curves.

\section{Examples}

We now consider two examples to illustrate the described technique. The first example is based on a recent meta-analysis of three case-control studies in Paget's disease [4]. In the second example, we consider the effect of a planned study on a current meta-analysis of 25 studies in human height [5], and illustrate the use of an additive genotype model as well as a quantitative phenotype.

\subsection{Example 1: Paget's Disease}

Chung et al. [4] present a recent meta-analysis of three case-control studies, in which they successfully detected a number of genetic associations with Paget's disease. We focus our attention here on one candidate SNP (rs825411) which did not reach the level of genome-wide significance $\left(\mathrm{p}<5.0 \times 10^{-8}\right)$ in their meta-analysis. This SNP was selected due to its location in the OPTN region, where there was previous evidence of a strong genetic signal [4, 14]. In this manner, we wish to address the question, if this observed association truly exists, how large a sample size would be required to achieve genome-wide significance in a meta-analysis of a newly planned study together with the three existing studies? A summary of the study-specific study characteristics, odds ratios and meta-analysis for rs 825411 is presented in table 2 . For the sake of simplicity, we consider an anticipated MAF of $49.8 \%$ for rs825411, as observed in the control group of the larger British cohort [14]. For reference, the specific case and control MAFs for each of the three studies are included in table 3, while a small sensitivity analysis explores the limiting MAF values of 0.46 and 0.60 and the effect of an unbalanced case-control design (table 4). The $I^{2}=60 \%$ indicates moderate heterogeneity in our analysis of the three studies.

The median $\mathrm{p}$ value of the updated meta-analysis, including the previously completed and the planned study, is presented in figure 1. In addition, a boxplot of the simulated $p$ values is included for five sample sizes of interest. These boxplots provide additional insight into the range of conclusions that could be observed from the updated meta-analysis. Moreover, note that the range of observed $\mathrm{p}$ values increases in conjunction with the planned study 


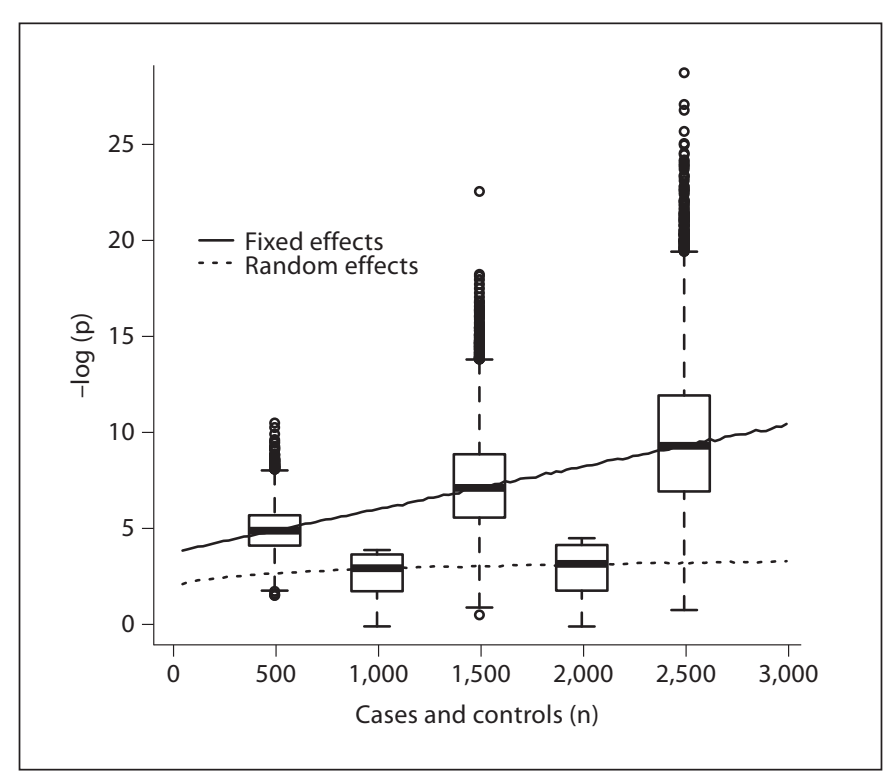

Fig. 1. The fixed effects meta-analysis (solid line) suggests that a study enrolling approximately 2,000 Paget cases and controls will likely detect a genome-wide association between rs825411 and Paget's disease. The random effects meta-analysis (dotted line) suggests that a study enrolling in excess of 3,000 cases and controls remains unable to detect a genome-wide association. Note the increased variation in $\mathrm{p}$ values in the fixed effects model, and that the dispersion of $\mathrm{p}$ value increases with the size of the planned study.

size, particularly in the fixed effects case. This is less evident in the random effects context, as a large study does not have as large of an impact on the updated meta-analysis due to the presence of heterogeneity.

Figure 1 shows that a planned study enrolling approximately 1,950 cases and controls will likely detect a statistically significant association $\left(<5.0 \times 10^{-8}\right)$ between rs 825411 and Paget's disease using the fixed effects model (fig. 1, solid line). In contrast to this result, the random effects meta-analysis (fig. 1, dotted line) suggests that even a large study will not provide evidence of a statistically significant association in the updated meta-analysis due to the large between-study heterogeneity parameter $\tau^{2}$. In fact, simulation results indicate that even an arbitrarily large study (in excess of 100,000 cases) will rarely produce an expected $\mathrm{p}$ value $<10^{-4}$. This is because the addition of a large study in the random effects model does not carry a large weight in the presence of heterogeneity. In light of this evidence, an investigator may conclude that unless a very large number of Paget cases are available, he is unlikely to obtain evidence of genome-wide significance between rs825411 and Paget's disease, and
Table 2. Meta-analysis of a candidate SNP for Paget's disease: rs825411

\begin{tabular}{lrcll}
\hline Study & Cases & Controls & OR $(95 \%$ CI $)$ & p value \\
\hline Belgian study $^{1}$ & 247 & 255 & $1.06(0.82,1.36)$ & 0.65 \\
$\begin{array}{l}\text { Dutch study } \\
\text { British replication }\end{array}$ & 79 & 95 & $1.60(1.07,2.41)$ & 0.025 \\
$\quad$ cohort & & & & \\
$\begin{array}{l}\text { Meta-analysis } \\
\quad \text { Fixed effects }\end{array}$ & 256 & 488 & $1.49(1.19,1.85)$ & 0.00030 \\
$\quad$ & & & $1.33(1.14,1.55)$ & 0.00026 \\
$\quad$ Random effects & & & $1.34(1.04,1.73)$ & 0.024
\end{tabular}

Note that $I^{2}=60 \%$, indicating moderate heterogeneity. The $\mathrm{p}$ value from the Breslow-Day test for heterogeneity is 0.062 .

${ }^{1}$ From Chung et al. [4]. ${ }^{2}$ From Albagha et al. [14].

Table 3. Meta-analysis of rs825411: MAF

\begin{tabular}{llll}
\hline Study & \multicolumn{2}{l}{ MAF } & \\
\cline { 2 - 4 } & cases & controls & $\begin{array}{l}\text { weighted } \\
\text { total }\end{array}$ \\
\hline Belgian study $^{1}$ & 0.56 & 0.55 & 0.55 \\
Dutch study $^{1}$ & 0.60 & 0.47 & 0.53 \\
British replication cohort $^{2}$ & 0.60 & 0.50 & 0.53 \\
\hline
\end{tabular}

MAF values are presented with $\mathrm{C}$ as the risk allele. Calculations are performed with the precision of the original papers.

${ }^{1}$ From Chung et al. [4]. ${ }^{2}$ From Albagha et al. [14].

Table 4. Approximate number of subjects required for a median p value $<5.0 \times 10^{-8}$ under various assumptions for rs 825411

\begin{tabular}{llll}
\hline $\begin{array}{l}\text { Ratio of cases } \\
\text { to controls }\end{array}$ & MAF & \\
\cline { 2 - 4 } & 0.46 & 0.50 & 0.60 \\
\hline $1: 1$ & 3,900 & 3,860 & 4,250 \\
$2: 3$ & 4,200 & 4,150 & 4,460 \\
$1: 2$ & 4,420 & 4,400 & 4,800 \\
$1: 3$ & 5,400 & 5,360 & 5,860 \\
\hline
\end{tabular}

even then, only in the fixed effects case. For this reason, future investigation of this SNP may not be an appropriate use of resources.

The sensitivity analysis for a fixed effects meta-analysis (table 4) illustrates the impact of MAF frequency and an unbalanced case-control ratio. Note that this design is optimal when the number of cases and controls is equal; 
Table 5. Meta-analysis of height SNPs

\begin{tabular}{llllll}
\hline SNP & MAF & \multicolumn{2}{l}{ Phase I } & & \\
\cline { 3 - 6 } & & effect & $\mathrm{SE}^{1}$ & $\mathrm{p}$ & $I^{2}, \%$ \\
\hline rs3796529 & 0.19 & 0.26 & 0.053 & $5.1 \times 10^{-7}$ & 32 \\
rs2516448 & 0.49 & 0.21 & 0.063 & $9.3 \times 10^{-4}$ & 0 \\
\hline
\end{tabular}

$\mathrm{SE}=$ Standard error.

${ }^{1}$ Standard errors were calculated from the inverse of a standard normal distribution and the published $\mathrm{p}$ value.

Table 6. Hardy-Weinberg proportions for fixed MAFs

\begin{tabular}{llll}
\hline Genotype & Proportion & rs3796529 & rs2516448 \\
\hline 0 & $P_{0}=(1-\mathrm{p})^{2}$ & $0.6561 n$ & $0.2601 n$ \\
1 & $P_{1}=2 \mathrm{p}(1-\mathrm{p})$ & $0.3078 n$ & $0.4997 n$ \\
2 & $P_{2}=\mathrm{p}^{2}$ & $0.0361 n$ & $0.2401 n$ \\
\hline MAF & & 0.19 & 0.49 \\
\hline
\end{tabular}

however, in the context of this example, the total required sample size is typically within $10-20 \%$ of this optimal case provided the ratio of cases to controls is no more than 1:2. Moreover, the capability to examine an unbalanced design and allow for uncertainty in MAF using the metaSNPPER package is a particular strength of the approach. Finally, as previously discussed, the random effects analysis does not provide evidence of a statistically significant genetic effect in any of these cases.

\subsection{Example 2: Human Adult Stature}

As a second example, we consider a recent report [5] of summary information for 42 loci associated with height in a meta-analytic framework based on participants of European ancestry. The meta-analysis was performed in four phases, where phase I included a meta-analysis of 25 studies (53,394 individuals) based on individual-level data. Specifically, we examine the plausibility of showing genome-wide significance $\left(\mathrm{p}<5 \times 10^{-8}\right)$ for two SNPs (rs3796529 and rs2516448) in the fixed effects meta-analysis (table 5). These SNPs were chosen as they are the highest ranking SNPs from table 2 of Lanktree et al. [5] that did not reach genome-wide significance. As height is a continuous trait, we focus attention on the difference in $\mathrm{cm}$ per allele as the genetic association measure of interest. Note that between-study heterogeneity appears minimal due to the relatively low values of $I^{2}$ for each of these two SNPs (0 and 32\%, respectively) [10]. Furthermore, in this example, we initially fix MAF for each SNP at 0.19 and 0.49 , respectively. Following this, we consider the impact of introducing additional uncertainty in the MAF by sampling the potential MAF from two uniform distributions, centered around the values of 0.19 and 0.49 , respectively (section 2.4). With this information, we proceed with an extension of steps 3 through 5 of the previously described summary (table 1).

In step 3, we sample the anticipated effect measure for the planned study from a normal distribution centered around the meta-analytic effect size. For the case of rs3796529, an estimate of the anticipated allele effect $\beta$ is centered around 0.26 with variance, $0.053^{2}$. This standard error, reported in table 5, corresponds to the metaanalytic linear regression coefficient, and is expected to be a suitable estimate of the uncertainty in the expected regression coefficient in the new study. Assuming the population is in Hardy-Weinberg equilibrium, a planned genetic study enrolling $n$ participants will have approximately the genotype frequencies provided in table 6 .

For step 4 (adapted to this quantitative trait), consider the genotype score $X_{i}$ for the $i$-th participant in the newly planned study. For this participant, generate a uniform $(0,1)$ random variable $(z)$ to determine the genotype score $\left(X_{i}\right)$ of this individual. If $z$ is $<P_{0}$ assign the genotype score 0 , if $P_{0}<z<P_{0}+P_{1}$ assign score 1 and if $P_{0}+P_{1}<$ $z<1$ assign the genotype score 2 . This ensures random variation in the study population of the planned study. Note that when the MAF is simulated from a uniform distribution, the bounds of $P_{0}, P_{1}$ and $P_{2}$ change with each iteration of the algorithm.

In the 5th step, generate individual-level data according to the predicted effect size (step 3) in the planned study and the genotype score (step 4). That is, in the case of rs3796529, $Y_{i}$ is sampled from a normal distribution with mean, $\beta X_{i}$ and variance $0.053^{2}$. Note that we do not incorporate any additional covariates, as we adopt the simplifying assumption that all necessary confounders (e.g. age) are appropriately accounted for in the initial meta-analysis. These $n$ simulated values can now be used to obtain the linear regression coefficient $(\beta)$ as an estimate of the genetic effect for the planned study (step 6) and the resulting value can be re-meta-analyzed using the fixed effects model (step 7).

As a technical note, since we do not have access to the summary effect measures for each study in the published report [5], we use algebraic manipulation to determine the effect measure and its variance upon adding a new study to the phase I meta-analysis. Let $\hat{\beta}_{F}^{0}$ denote the 


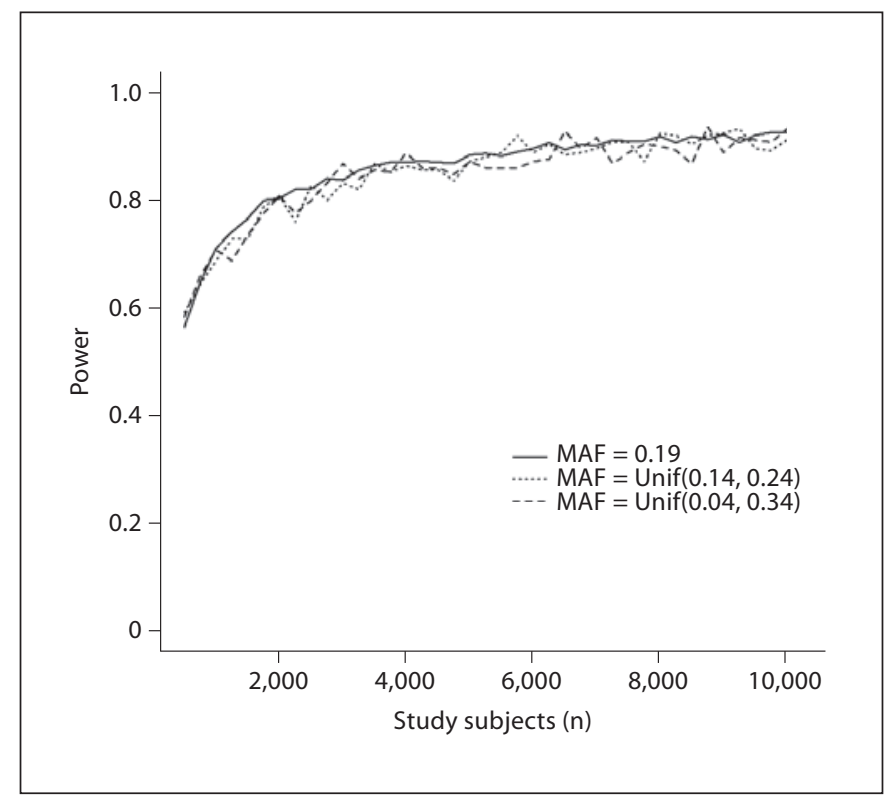

Fig. 2. The empirical power of the updated fixed effects meta-analysis to detect genome-wide significance between rs3796529 and height for a fixed value of the MAF of 0.19 and two uniform distributions. Note the increased oscillation (uncertainty) that the prior distributions introduce in the empirical estimation of power.

fixed effects estimate from the initial $K=25$ studies with corresponding variance $\operatorname{Var}\left(\hat{\beta}_{F}^{0}\right)$. It can be shown (Appendix) that upon adding the new study, the updated effect measure, $\hat{\beta}_{F}^{1}$, may be represented as:

$$
\hat{\beta}_{F}^{1}=\frac{\frac{\hat{\beta}_{F}^{0}}{\operatorname{Var}\left(\hat{\beta}_{F}^{0}\right)}+\omega_{K+1} \beta_{K+1}}{\frac{1}{\operatorname{Var}\left(\hat{\beta}_{F}^{0}\right)}+\omega_{K+1}}
$$

and, its variance, $\operatorname{Var}\left(\hat{\beta}_{F}^{1}\right)$ may be calculated from:

$$
\operatorname{Var}\left(\hat{\beta}_{F}^{1}\right)=\frac{1}{\frac{1}{\operatorname{Var}\left(\hat{\beta}_{F}^{0}\right)}+\omega_{K+1}}
$$

Thus, we do not require the effect measures in each study in the fixed effects meta-analysis case. The entire procedure is then replicated 10,000 times, and then repeated for a new value of $n$. Note that this algebraic manipulation would not apply for a random effects metaanalysis, as the introduction of the new study would lead to a revised estimate of $\tau^{2}$ and revised study weights.

Cumulative Meta-Analyses and Sample Size

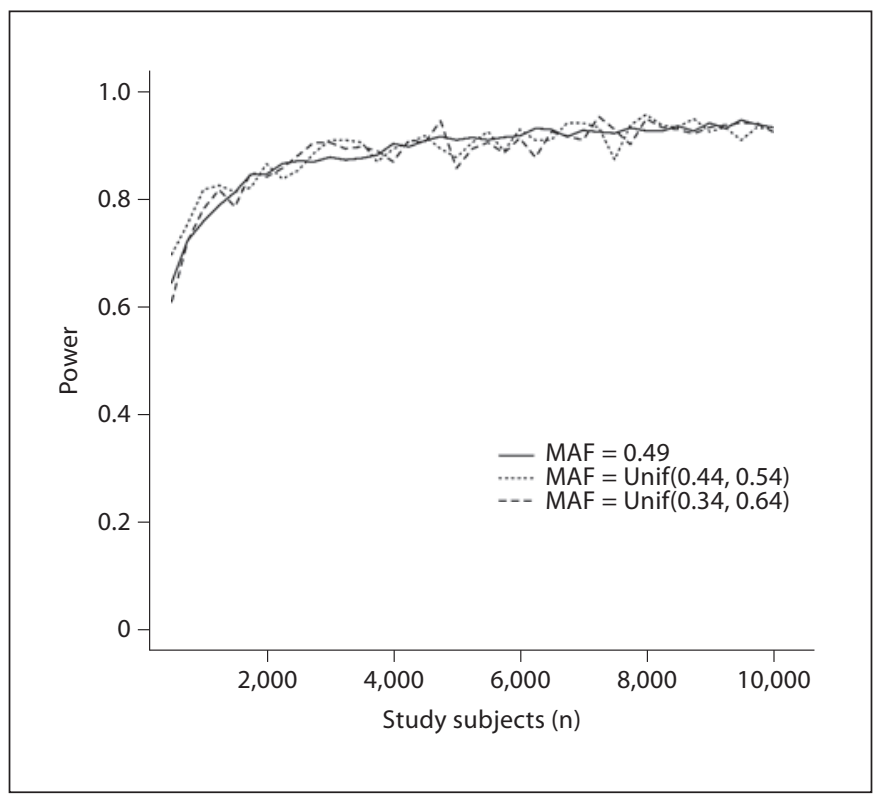

Fig. 3. The empirical power of the updated meta-analysis to detect genome-wide significance between rs2516448 and height for a fixed value of the MAF of 0.19 and two uniform distributions on various ranges. Note the increased uncertainty as the plausible range of the MAF increases.

From this analysis, it appears that a study enrolling approximately 2,000 subjects is likely to have approximately $80 \%$ power to detect the association at the genome-wide significance level for rs 3796529 should this association be true assuming a fixed MAF of 0.19 . However, if additional uncertainty is included through the use of a uniform distribution for the MAF, the required sample size increases to approximately 2,500 participants (fig. 2). Likewise, a total of 2,000 subjects is required for the detection of a statistically significant effect of rs 2516448 using a fixed MAF of 0.49 , as well as both MAF distributions. Note that in this case, the use of the uniform distribution with a similar range length has less of an impact on the observed power due to our proximity to 0.50 (fig. 3). As such, in combination with existing data, a new study enrolling approximately 2,500 participants will likely be sufficiently powered to detect both associations.

\section{Discussion}

The evidence-based algorithm we describe provides an empirical estimate of the impact of a newly planned study on a cumulative meta-analysis incorporating the 
planned study. This approach may be used to inform sample size to better detect possible genetic candidates, when the results of the planned study will be combined with other analyses. Given the relatively small effect sizes that are observed in genetic associations, the described technique can be used to supplement traditional sample size estimation approaches [15] and software (e.g. Quanto [16]), which do not consider the impact of the planned study in conjunction with existing meta-analytic results. Note that the approach only requires a reasonable estimate of the anticipated effect size and its variance (ideally from a current meta-analysis of studies), and an estimate of the MAF (or a reasonable range).

The proposed algorithm can also be compared with other cumulative meta-analysis techniques in the literature. For example, Barrowman et al. [17] provide a formula to estimate the number of participants that must be enrolled in a new study to obtain statistical significance. In the context of a genetic study, as statistical significance requires an exceptionally small $\mathrm{p}$ value, this approach suggests rather large sample sizes. For example, in excess of 65,000 additional subjects would be required in the height example for rs3796529. In addition, Bagos and Nikolopoulos [18] provide a regression-based approach, which may be used to detect whether the magnitude of the effect size changes with an increasing number of studies in the meta-analysis, while formal stopping rules to control for multiple testing in cumulative meta-analyses are advocated in Higgins et al. [19].

In their consideration of power in one-stage and twostage genome-wide analysis, Chapman et al. [20] comprehensively examine sample size requirements under a fixed effects model across a large number of combinations of odds ratios and MAF in a 1:1 case-control design, assuming homogeneity between stages. Their approach allows an investigator to assess power for all SNPs of potential interest in the context of a genomewide scan, without knowledge of the effect sizes and allele frequencies that will be observed when the study is carried out. In contrast, the method we propose incorporates information about effect size estimates and standard errors from each of the existing studies available for meta-analysis. In principle, our approach could be applied to every SNP in an existing GWAS meta-analysis and the genome-wide power distribution determined for the updated meta-analysis, e.g. how many SNPs would have expected power $>50$ or $>80 \%$ when a new study with a given sample size is added. We anticipate, however, that the method will be most useful when examining a set of prespecified SNPs of interest (e.g. the top-ranked SNPs that did not reach genome-wide significance). In particular, our approach produces substantially more detail for specific SNPs of interest through the use of unbalanced case-control designs, variability in specification of the distribution of the MAF, direct examination of $p$ values, and incorporation of the random effects model.

The approach is thus presented in a gene discovery context, suggesting future candidate SNPs that may be tested in subsequent validation studies. In this manner, a meta-analysis of observational studies will rarely serve as the sole basis for determining whether a locus is associated with a trait of interest but rather represents an early stage of scientific discovery. This is in sharp contrast with the role of meta-analysis in the context of randomized trials, where the results of a review by the Cochrane Collaboration [21] may directly and immediately inform clinical practice.

Other uses of the proposed algorithm may include design of two-stage or replication studies [22, 23]. However, as presented in this simple form, the method does not adjust for sequential testing in the algorithm, so there is an increased risk of an inflated type I error rate. We thus recommend use of the algorithm to assist in the design of additional data collection to detect SNPs that are 'nearly' significant at the genome-wide level in an existing metaanalysis. In practice, we suggest that an investigator would prioritize high-ranking SNPs that did not reach the threshold of genome-wide significance for further investigation in a meta-analytic framework. Note that the number of SNPs to investigate is entirely at the researcher's discretion, and limited only by the requirement to specify relevant parameters and by the quantity of information that our technique may produce. Alternatively, the algorithm may be applied to assess the potential futility of a small genetic study. Finally, note that metaSNPPER can examine statistical significance at any threshold level of interest, which may be of use in a candidate gene study, which requires smaller thresholds of statistical evidence.

There are some important considerations to be made when applying the algorithm: firstly, the prescribed algorithm makes no allowance for the 'winner's curse' [24], whereby the reported effect sizes may be artificially inflated. However, recent approaches $[25,26]$ could easily be applied to the preliminary estimate of the effect measure and its variance. In this modified framework, one would then simulate the effect size from these biased-reduced estimates and confidence intervals. Note that larger standard deviations or, similarly, smaller genetic effect 
sizes may also be explored in a sensitivity analysis should the investigator feel the anticipated standard deviation and/or effect size is overly optimistic.

Secondly, the role of heterogeneity in the effect measures as well as population stratification may be of concern. In particular, population heterogeneity or differences in environmental factors may reduce the validity of combining the studies in a meta-analysis, as the observed effect measures may exhibit systematic differences. In particular, care must be taken when applying this approach to trans-ethnic meta-analyses, as significant heterogeneity may be due to differences in the genetic effects of these distinct groups. However, these weaknesses are not unique to the algorithm and apply to meta-analysis of genetic association studies and observational studies in general. The method is most useful to provide an estimate of power in cases where heterogeneity prohibits the use of the fixed effects model; however, the genetic effects are still sufficiently similar to proceed with a pooled analysis. This is of particular interest as estimation of power in the random effects meta-analysis is less straightforward.

In the case of known effect modifiers or covariates of interest, a similar procedure could also be applied to a meta-analysis of interaction terms [27]. While this approach may suffer from complications associated with lack of data availability, it may prove suitable in a consortium setting when data are shared in a standardized manner.

Finally, recent attention to the potential efficiency of meta-analysis as compared to individual-level analyses in certain situations $[7,28]$ provides a statistical justification for the use of pooled analysis techniques in practice. Specifically, provided the genetic associations are similar across studies, a meta-analysis of summary statistics can be as efficient as an analysis using individual-level data in both logistic and linear regression settings [28].

\section{Acknowledgements}

M.A.R. is supported by a Natural Sciences and Engineering Research Council of Canada (NSERC) Post-Doctoral Fellowship. S.B.B. is supported by NSERC and the Canadian Institutes of Health Research. The authors thank Andrew Paterson for helpful discussion and suggestions.

\section{Appendix}

Let $\hat{\beta}_{F}^{0}$ denote the fixed effects estimate from the initial $K$ studies with corresponding variance $\operatorname{Var}\left(\hat{\beta}_{F}^{0}\right)$. Upon adding the additional study, the new effect measure, $\hat{\beta}_{F}^{1}$, may be represented as:

$$
\begin{aligned}
\hat{\beta}_{F}^{1}= & \frac{\sum_{k=1}^{K+1} \omega_{k} \hat{\beta}_{k}}{\sum_{k=1}^{K+1} \omega_{k}} \\
= & \frac{\sum_{k=1}^{K} \omega_{k} \hat{\beta}_{k}+\omega_{K+1} \hat{\beta}_{K+1}}{\sum_{k=1}^{K} \omega_{k}+\omega_{K+1}} \\
= & \frac{\frac{\hat{\beta}_{F}^{0}}{\operatorname{Var}\left(\hat{\beta}_{F}^{0}\right)}+\omega_{K+1} \hat{\beta}_{K+1}}{\frac{1}{\operatorname{Var}\left(\hat{\beta}_{F}^{0}\right)}+\omega_{K+1}}
\end{aligned}
$$

and, its variance, $\operatorname{Var}\left(\hat{\beta}_{F}^{1}\right)$ may be calculated from:

$$
\begin{aligned}
\operatorname{Var}\left(\hat{\beta}_{F}^{1}\right) & =\frac{1}{\sum_{k=1}^{K+1} \omega_{k}} \\
& =\frac{1}{\sum_{k=1}^{K} \omega_{k}+\omega_{K+1}} \\
& =\frac{1}{\frac{1}{\operatorname{Var}\left(\hat{\beta}_{F}^{0}\right)}+\omega_{K+1}}
\end{aligned}
$$

\section{References}

1 Cornelis MC, Agrawal A, Cole JW, Hansel $\mathrm{NN}$, Barnes KC, Beaty TH, Bennett SN, Bierut LJ, Boerwinkle E, Doheny KF, et al; GENEVA Consortium: The Gene, Environment Association Studies consortium (GENEVA): maximizing the knowledge obtained from GWAS by collaboration across studies of multiple conditions. Genet Epidemiol 2010;34:364-372.

-2 Sutton AJ, Cooper NJ, Jones DR, Lambert PC, Thompson JR, Abrams KR: Evidence-

Cumulative Meta-Analyses and Sample Size based sample size calculations based upon updated meta-analysis. Stat Med 2007;26: 2479-2500.

3 Rotondi M, Donner A: Sample size estimation in cluster randomized trials: an evidence-based perspective. Comp Stat Data Anal 2012;56:1174-1187.

-4 Chung PY, Beyens G, Boonen S, Papapoulos S, Geusens P, Karperien M, Vanhoenacker F, Verbruggen L, Fransen E, Van Offel J, et al: The majority of the genetic risk for Paget's disease of bone is explained by genetic variants close to the CSF1, OPTN, TM7SF4, and TNFRSF11A genes. Hum Genet 2010;128: 615-626.

5 Lanktree MB, Guo Y, Murtaza M, Glessner JT, Bailey SD, Onland-Moret NC, Lettre G, Ongen H, Rajagopalan R, Johnson T, et al: Meta-analysis of dense genecentric association studies reveals common and uncommon variants associated with height. Am J Hum Genet 2011;88:6-18. 
6 R Development Core Team: R: A Language and Environment for Statistical Computing. Vienna, R Foundation for Statistical Computing, 2012.

7 Lin DY, Zeng D: Meta-analysis of genomewide association studies: no efficiency gain in using individual participant data. Genet Epidemiol 2010;34:60-66.

8 DerSimonian R, Laird N: Meta-analysis in clinical trials. Cont Clin Trials 1986;7:177188 .

9 Higgins JPT, Thompson SG, Spiegelhalter DJ: A re-evaluation of random-effects metaanalysis. J R Stat Soc Ser A Stat Soc 2009;172: $138-159$.

10 Higgins JP, Thompson SG: Quantifying heterogeneity in a meta-analysis. Stat Med 2002; 21:1539-1558.

-11 Dudbridge F, Gusnanto A: Estimation of significance thresholds for genomewide association scans. Genet Epidemiol 2008;32:227234.

12 Turner RM, Omar RZ, Thompson SG: Bayesian methods of analysis for cluster randomized trials with binary outcome data. Stat Med 2001;20:453-472.

13 Rotondi M, Donner A: Sample size estimation in cluster randomized educational trials: an empirical Bayes approach. J Educ Behav Stat 2009;34:229-237.

14 Albagha OME, Visconti MR, Alonso N, Langston AL, Cundy T, Dargie R, Dunlop MG, Fraser WD, Hooper MJ, Isaia G, et al: Genome-wide association study identifies variants at CSF1, OPTN and TNFRSF11A as genetic risk factors for Paget's disease of bone. Nat Genet 2010;42:520-524.
15 Spencer CC, Su Z, Donnelly P, Marchini J: Designing genome-wide association studies: sample size, power, imputation, and the choice of genotyping chip. PLoS Genet 2009; 5:e1000477.

16 Gauderman W, Morrison J: QUANTO 1.1: A computer program for power and sample size calculations for genetic-epidemiology studies, 2006.

17 Barrowman N, Fang M, Sampson M, Moher $\mathrm{D}$ : Identifying null meta-analyses that are ripe for updating. BMC Med Res Meth 2003; 3:13.

18 Bagos P, Nikolopoulos G: Generalized least squares for assessing trends in cumulative meta-analysis with applications in genetic epidemiology. J Clin Epidem 2009;62:10371044.

19 Higgins J, Whitehead A, Simmonds M: Sequential methods for random-effects metaanalysis. Stat Med 2011;30:903-921.

-20 Chapman K, Ferreira T, Morris A, Asimit J, Zeggini E: Defining the power limits of genome-wide association scan meta-analyses. Genet Epidemiol 2011;35:781-789.

21 The Cochrane Collaboration. Oxford, UK, 2012. http://www.cochrane.org/ (accessed April 5, 2012).

22 Thomas D, Casey G, Conti DV, Haile RW, Lewinger JP, Stram DO: Methodological issues in multistage genome-wide association studies. Stat Sci 2009;24:414-429.

23 Skol AD, Scott LJ, Abecasis GR, Boehnke M: Joint analysis is more efficient than replication-based analysis for two-stage genomewide association studies. Nat Genet 2006;38: 209-213.
24 Garner C: Upward bias in odds ratio estimates from genome-wide association studies. Genet Epidemiol 2007;31:288-295.

25 Ghosh A, Zou F, Wright FA: Estimating odds ratios in genome scans: an approximate conditional likelihood approach. Am J Hum Genet 2008;82:1064-1074.

26 Sun L, Dimitromanolakis A, Faye LL, Paterson $\mathrm{AD}$, Waggott $\mathrm{D}$, Bull SB: BR-squared: a practical solution to the winners curse in genome-wide scans. Hum Genet 2011;129: 545-552.

27 Manning AK, LaValley M, Liu CT, Rice K, An P, Liu Y, Miljkovic I, Rasmussen-Torvik L, Harris TB, Province MA, et al: Meta-analysis of gene-environment interaction: joint estimation of SNP and SNP $\times$ environment regression coefficients. Genet Epidem 2011; 35:11-18.

28 Lin DY, Zeng D: On the relative efficiency of using summary statistics versus individuallevel data in meta-analysis. Biometrika 2010; 97:321-332.

29 Frazer KA, Ballinger DG, Cox DR, Hinds DA, Stuve LL, Gibbs RA, Belmont JW, Boudreau A, Hardenbol P, Leal SM, et al: A second generation human haplotype map of over 3.1 million SNPs. Nature 2007;449:851861.

30 Durbin RM, Altshuler DL, Abecasis GR, Bentley DR, Chakravarti A, Clark AG, Collins FS, De La Vega FM, Donnelly P, Egholm $\mathrm{M}$, et al: A map of human genome variation from population-scale sequencing. Nature 2010;467:1061-1073. 